\title{
Effects of Cholecalciferol on Key Components of Vitamin D-Endo/ Para/Autocrine System in Experimental Type 1 Diabetes
}

\author{
Anna Mazanova $\left(\mathbb{D},{ }^{1}\right.$ Ihor Shymanskyi ${ }^{1},{ }^{1}$ Olha Lisakovska, ${ }^{1}$ Lala Hajiyeva, ${ }^{2}$ \\ Yulia Komisarenko, ${ }^{2}$ and Mykola Veliky ${ }^{1}$ \\ ${ }^{1}$ Department of Biochemistry of Vitamins and Coenzymes, Palladin Institute of Biochemistry of the National Academy of Sciences of \\ Ukraine, Kyiv, Ukraine \\ ${ }^{2}$ Department of Endocrinology, Bogomolets National Medical University, Kyiv, Ukraine
}

Correspondence should be addressed to Anna Mazanova; ann.mazanova@gmail.com

Received 25 August 2017; Revised 2 December 2017; Accepted 12 December 2017; Published 6 February 2018

Academic Editor: Michael Horowitz

Copyright (c) 2018 Anna Mazanova et al. This is an open access article distributed under the Creative Commons Attribution License, which permits unrestricted use, distribution, and reproduction in any medium, provided the original work is properly cited.

\begin{abstract}
Objectives. Recent prospective studies have found the associations between type 1 diabetes (T1D) and vitamin D deficiency. We investigated the role of vitamin $\mathrm{D}$ in the regulation of $25 \mathrm{OHD}-1 \alpha$-hydroxylase (CYP27B1) and VDR expression in different tissues of T1D rats. Design. T1D was induced in male Wistar rats by streptozotocin $(55 \mathrm{mg} / \mathrm{k}$ b.w.). After 2 weeks of T1D, the animals were treated orally with or without vitamin $\mathrm{D}_{3}$ (cholecalciferol; $100 \mathrm{IU} / \mathrm{rat}, 30$ days). Methods. Serum 25hydroxyvitamin D (25OHD) was detected by ELISA. CYP27A1, CYP2R1, CYP27B1, and VDR were assayed by RT-qPCR and Western blotting or visualized by immunofluorescence staining. Results. We demonstrated that T1D led to a decrease in blood $25 \mathrm{OHD}$, which is probably due to the established downregulation of CYP27A1 and CYP2R1 expression. Vitamin D deficiency was accompanied by elevated synthesis of renal CYP27B1 and VDR. Conversely, CYP27B1 and VDR expression decreased in the liver, bone tissue, and bone marrow. Cholecalciferol administration countered the impairments of the vitamin D-endo/para/ autocrine system in the kidneys and extrarenal tissues of diabetic rats. Conclusions. T1D-induced vitamin D deficiency is associated with impairments of renal and extrarenal CYP27B1 and VDR expression. Cholecalciferol can be effective in the amelioration of diabetes-associated abnormalities in the vitamin D-endo/para/autocrine system.
\end{abstract}

\section{Introduction}

Vitamins of $\mathrm{D}$ group (predominantly vitamin $\mathrm{D}_{2}$ and $\mathrm{D}_{3}$ ) are biologically active substances of secosteroid nature [1]. The effects of vitamin $\mathrm{D}$, which go beyond regulation of calcium homeostasis and bone remodeling, have currently received much attention. Recent evidence suggests that hormonally active form of vitamin D, $1,25(\mathrm{OH})_{2} \mathrm{D}$ (calcitriol), is able to regulate important cellular processes such as proliferation and differentiation, immune responses, osteoimmunity, angiogenesis, and apoptosis [2, 3]. Vitamin D deficiency and disruptions in the vitamin $\mathrm{D}$-endo/para/autocrine system can increase the risk of autoimmune diseases, in particular type 1 diabetes [4]. Several studies have shown that vitamin D plays an important role in preventing pancreatic $\beta$-cell autoimmune destruction [5]. Calcitriol is reported to be directly involved in insulin production and can improve the sensitivity of peripheral tissues to insulin action [6].

The realization of the biological effects of vitamin D in cells is closely related to the functioning of the vitamin D-endo/para/autocrine system, which includes (1) photoconversion of 7-dehydrocholesterol in the skin with the formation of cholecalciferol; (2) synthesis of 25OHD (calcidiol) in the liver by means of two key vitamin D 25hydroxylases (cytochromes P450), CYP2R1 and CYP27A1; (3) conversion of 25 -hydroxyvitamin $\mathrm{D}(25 \mathrm{OHD})$ in the kidneys or extrarenal tissues to hormonally active form, $1 \alpha, 25(\mathrm{OH})_{2} \mathrm{D}$, by $25 \mathrm{OH}-1 \alpha$-hydroxylase (CYP27B1); (4) calcitriol transport to target organs by vitamin $\mathrm{D}$ binding 
protein (VDBP); and (5) binding of $1 \alpha, 25(\mathrm{OH})_{2} \mathrm{D}$ to vitamin $\mathrm{D}$ receptors (VDR) and regulation of gene expression [7].

While renal activation of CYP27B1 leads to the formation of the vitamin $\mathrm{D}$ hormone, $1,25(\mathrm{OH})_{2} \mathrm{D}$, which is mainly responsible for the calcium absorption in the intestine and kidneys, extrarenal calcitriol covers a wider range of noncalcemic physiological functions $[2,3]$. There are different cell types that express CYP27B1, such as cutaneous and intestinal epithelial cells, macrophages, and breast and bone cells [8]. The physiological and pharmacological effects of $1,25(\mathrm{OH})_{2} \mathrm{D}$ are mediated by VDR, the member of the superfamily of steroid nuclear receptors [2]. Being inherent to a variety of target cells, these receptors provide a significant therapeutic potential of vitamin D as a natural VDR ligand in many diseases.

The relationship between the bioavailability of vitamin $\mathrm{D}$, its metabolism, and T1D development is multifaceted and is confirmed by genetic studies in humans. It has been established that $25 \mathrm{OHD}$ deficiency, associated with cytochrome polymorphism and inherited defects in vitamin D metabolism, predisposes to T1D and its complications. In particular, there is a close linkage between the polymorphism of certain alleles of Cyp2r1 gene, decreased level of circulating $25 \mathrm{OHD}$, and the development of T1D as well as diabetes-associated secondary osteoporosis [9]. The importance of the vitamin D-endo/para/autocrine system is further confirmed by the correlation between T1D development, osteoporosis, and the presence of functionally defective alleles in Cyp27b1 and $V d r$ genes $[10,11]$. Consequently, abnormal activity of vitamin $\mathrm{D}$ hydroxylation enzymes and inadequacy of VDR for a correct signal transduction may have relevance to $\mathrm{T} 1 \mathrm{D}$ and its complications.

In chronic diabetes, a number of abnormalities are observed in tissues that potentially participate in vitamin D metabolism. However, there is no unambiguous evidence for TD1-associated expression of CYP27B1 and VDR, precisely those components that link vitamin $\mathrm{D}$ metabolism and signaling. Therefore, our aim was to investigate how T1D changes tissue distribution of CYP27B1 and VDR and whether vitamin $\mathrm{D}_{3}$ (cholecalciferol) treatment can affect diabetes-related dysfunction of the vitamin D-endo/para/ autocrine system.

\section{Materials and Methods}

2.1. Experimental Design. Male Wistar rats $(140 \pm 7 \mathrm{~g})$ were injected with streptozotocin at dose $55 \mathrm{mg} / \mathrm{kg}$ of b.w. Control animals received $10 \mathrm{mM}$ citrate buffer solution. After two weeks of diabetes induction, the animals were divided into two groups: diabetic rats and diabetic rats, which were treated per os with $100 \mathrm{IU}$ of vitamin $\mathrm{D}_{3}$ for 30 days. All animal procedures were performed in accordance with the European Convention for Protection of Vertebrate Animals used for Research and Scientific Purposes (Strasbourg, 1986), General Ethical Principles of Animal Experimentation, approved by the First National Congress on Bioethics (Kyiv, 2001).
2.2. Serum $250 H D$. The content of $25 \mathrm{OHD}$ in the blood serum was determined by the in-house developed ELISA kit [12].

\subsection{Isolation of Bone Marrow Cells and Immunocytochemistry.} The bone marrow cells were obtained according to protocol, which we described previously [13]. For fluorescence visualization, we used primary antibody to CYP27B1 and VDR (1:200; Santa Cruz Biotech., USA) and secondary antibodies DyLight 488 and Alexa Fluor 546 (1:250; ThermoFisher, USA). Nuclei were stained with Hoechst $(0.1 \mu \mathrm{g} / \mathrm{mL}$; Sigma, USA). Samples were analyzed on a LSM 510 META confocal microscope (100x magnification).

2.4. SDS-PAG Electrophoresis and Western Blotting (WB). Equal protein samples $(50 \mu \mathrm{g})$ were separated by $10 \%$ SDSPAGE and then transferred onto nitrocellulose membranes. For protein signal detection, we used primary antibodies against VDR, CYP27B1 ( $1: 200$; Santa Cruz Biotech., USA), $\beta$-actin (1:25000; Sigma, USA), and secondary antibodies: anti-mouse (1:2500; Sigma, USA) and anti-goat (1:1000; Invitrogen, USA) conjugated with HRP. Membranes were subjected to chemiluminescent detection with p-coumaric acid (Sigma, USA) and luminol (AppliChem $\mathrm{GmbH}$, Germany). The immunoreactive bands were quantified with GelPro Analyzer 3.2.

2.5. RNA Extraction and Quantitative Real-Time Polymerase Chain Reaction (RT-qPCR). Total RNA was extracted with a mini PREP RNA mini kit (Qiagen, USA) according to the manufacturer's protocol. Quantity and purity of the RNA were determined by NanoDrop DeNovix DS-11. Synthesis of cDNA was carried out using a Maxima $\mathrm{H}$ Minus First Strand cDNA Synthesis Kit (ThermoFisher, USA) as described by the manufacturer. RT-qPCR was performed with Maxima SYBER Green/ROX qPCR Master Mix $(2 \times)$ (ThermoFisher, USA) and specific primers against $V d r$ ( $5^{\prime}$-TCATCCCTACTGTGTCCCGT- ${ }^{\prime}$ sense, $5^{\prime}$-TGAGTG CTCCTTGGTTCGTG-3' antisense), Cyp27a1 (5'-TCGA CACATCCTGATTGGAAGG-3' sense, $5^{\prime}$-TCTCATGCG GCTCAACACAG-3' antisense), Cyp2r1 (5'-CCTTCT GCTACTACTCGTGC-3' sense, $5^{\prime}$-GCATGGTCTATCTC GCCAAA- $3^{\prime}$ antisense), Cyp27b1 (5'-TGGGTGCTGGG AACTAACCC- $3^{\prime}$ sense, $5^{\prime}$-TCGCAGACTGATTCCACCT C-3' antisense), and Gapdh (5'-TGAACGGGAAGCTC ACTGG-3' sense, 5' ${ }^{\prime}$-TCCACCACCCTGTTGCTGTA-3' antisense). Data were calculated using the $\Delta \Delta C_{t}$ method.

2.6. Statistical Analysis. All results are expressed as mean \pm SEM. The Kolmogorov-Smirnov test was used for testing on normal distribution. Statistical differences between the various groups were compared by using the ANOVA test. Differences were considered significant when $p \leq 0.05$. All statistical analysis was performed using Origin Pro 8.5 (OriginLab Corporation, Northampton, MA, USA). 
TABle 1: Glucose and 25OHD levels at 6-week postinitiation of diabetes and after vitamin $\mathrm{D}_{3}$ treatment.

\begin{tabular}{lcc}
\hline & $\begin{array}{c}\text { Whole blood glucose } \\
\text { level, } \mathrm{mmol} / \mathrm{L}\end{array}$ & $\begin{array}{c}\text { Blood serum 25OHD } \\
\text { level, nmol/L }\end{array}$ \\
\hline Control & $4.9 \pm 0.2$ & $97.5 \pm 5.2$ \\
Diabetes & $26.8 \pm 2.9^{*}$ & $50.2 \pm 3.0^{*}$ \\
$\begin{array}{l}\text { Diabetes + 100 IU } \\
\text { vitamin } \mathrm{D}_{3}\end{array}$ & $21.7 \pm 2.5$ & $71.0 \pm 3.1^{\#}$ \\
\hline
\end{tabular}

Results are expressed as mean \pm SEM. ${ }^{*} p<0.05$ versus control; ${ }^{\#} p<0.05$ versus diabetes, $n=6$.

\section{Results}

We found that six weeks after the injection of streptozotocin, fasting blood glucose exceeds the control level by 5.5-fold $(p=0.0001)$ (Table 1). Glucose-lowering effect of cholecalciferol was statistically insignificant in diabetes. Chronic hyperglycemia was accompanied by profound changes in blood serum 25OHD content as a marker of vitamin D bioavailability. At week 6, after the initiation of diabetes, serum 25OHD levels were reduced by approximately $50 \%(p=0.0001)$ compared with controls (Table 1). Cholecalciferol administration during 30 days significantly improved vitamin D bioavailability.

Since the conversion of vitamin $\mathrm{D}$ to $25 \mathrm{OHD}$ predominantly catalyzes two cytochrome P450 isoforms (mitochondrial CYP27A1 and microsomal CYP2R1), it was useful to determine whether a significant 25OHD deficiency in diabetes is associated with any changes in the expression of these enzymes. It was shown that mRNA contents of Cyp27a1 and Cyp2r1 in the liver of diabetic rats decreased by 4.2 -fold $(p=0.039)$ and 6.3 -fold $(p=0.033)$, respectively, as compared with the control (Figure 1(a)). Diabetic rats administered with cholecalciferol demonstrated a 3.7 -fold increase in Cyp2r1 mRNA that corresponds to $60 \%$ of the control value $(p=0.033)$. There was no difference in the Cyp27a1 mRNA expression after vitamin $\mathrm{D}_{3}$ treatment compared with the diabetes group.

Next, we investigated the distribution of vitamin D-endo/ para/autocrine system components, such as CYP27B1 and VDR in different tissues. Their mRNA and protein levels were determined in classical (metabolic) tissues (liver and kidneys) and nonclassical tissues (bone and bone marrow). According to RT-qPCR data, the Cyp27b1 mRNA level in the liver of diabetic animals was significantly reduced (by 5.3-fold) as compared with the control $(p=0.035)$ (Figure 1(b)). Downregulation of cytochrome was further confirmed by WB (Figure 1(c)). Diabetic animals exhibited the CYP27B1 protein level 2.2-fold lower than in the control $(p=0.001)$. As shown in Figures $1(\mathrm{~d})$ and 1(e), similar changes in the expression of VDR at the transcriptional and translational levels were seen. The $V d r$ mRNA level was detected to be 14.3-fold less pronounced than in the control $(p=0.0004)$. As evidenced by the WB data, the VDR protein level in diabetes decreased 1.5-fold in comparison with the control $(p=0.009)$. Cholecalciferol treatment resulted in a 2.0 -fold $(p=0.035)$ and 5.7-fold $(p=0.0004)$ elevation of Cyp27b1 and Vdr mRNAs, respectively, compared with diabetic animals. While fully restoring the protein level of CYP27B1, cholecalciferol caused an increase in the protein VDR level that reached the value 2.2-fold higher compared with the control $(p=0.009)$.

Conversely, in the kidney tissue, there were opposite changes in the vitamin D-endocrine system. Vitamin Ddeficient status of the animals with T1D leads to an increase in CYP27B1 at both transcriptional (by 1.28 -fold, $p=0.003$ ) and translational (by 1.25-fold, $p=0.005$ ) levels in the renal tissue (Figures 2(a) and 2(b)). The same tendency, but more pronounced, was observed with respect to mRNA and protein content of VDR (Figures 2(c) and 2(d)). When measured by RT-qPCR, the level of $V d r$ mRNA in diabetic animals was 5.2 -fold higher than that in the control $(p=0.048)$, which was accompanied by a 2.0 -fold increase in the VDR protein level $(p=0.0002)$. As shown in Figure 2, the administration of cholecalciferol partially or completely abrogated the effects of diabetes on CYP27B1 and VDR expression in the kidneys.

It is known that the bones possess one of the most active vitamin D-para/autocrine systems. As shown in Figure 3(a), T1D caused a significant 13.0-fold elevation of Cyp27b1 mRNA in bone tissue $(p=0.006)$. Unexpectedly, WB revealed no significant changes in the synthesis of CYP27B1 protein (Figure 3(b)). Opposite results were observed for VDR expression in the bone tissue (Figures 3(c) and 3(d)), where a significant 3.3-fold reduction in $V d r$ mRNA was detected $(p=0.0003)$. Consistent with the diabetes-induced lowering of $V d r$ expression, there was a 1.8-fold decrease in the protein level of VDR in the bones $(p=0.0001)$. With a significant normalizing effect on $C y p 27 b 1$ gene transcription, cholecalciferol treatment increased CYP27B1 protein to a level twice that of the control. Vitamin $\mathrm{D}_{3}$ partially or completely corrected VDR expression in the bone tissue.

To further determine that altered CYP27B1 and VDR expression occurs in nonclassical tissues of diabetic animals, we isolated the bone marrow cells, which contain precursors of the main bone-forming cells, such as preosteoblasts and preosteoclasts. RT-qPCR study of isolated bone marrow cells demonstrated that in diabetes mRNA levels of Cyp27b1 (Figure 4) and $V d r$ (Figure 5) decreased 3.8 -fold $(p=0.0001)$ and 9.0-fold $(p=0.003)$, respectively, as compared with the control. These changes were further confirmed by decreased protein levels of CYP27B1 and VDR in the bone marrow cells, the expression of which was visualized using immunofluorescence staining and confocal microscopy. Vitamin $\mathrm{D}_{3}$ treatment led to partial or full normalization of the parameters studied.

\section{Discussion}

Type 1 diabetes is an autoimmune endocrine disease associated with $\beta$-cell destruction that leads to insulin insufficiency and chronic hyperglycemia [14]. In recent years, a number of mechanisms for islet autoimmunity have been proposed, one of which is the potential negative impact of vitamin $\mathrm{D}$ deficiency. The latest advances in the area of vitamin D biology have enabled us to better understand how vitamin $\mathrm{D}$ deficiency can be involved in T1D and its 

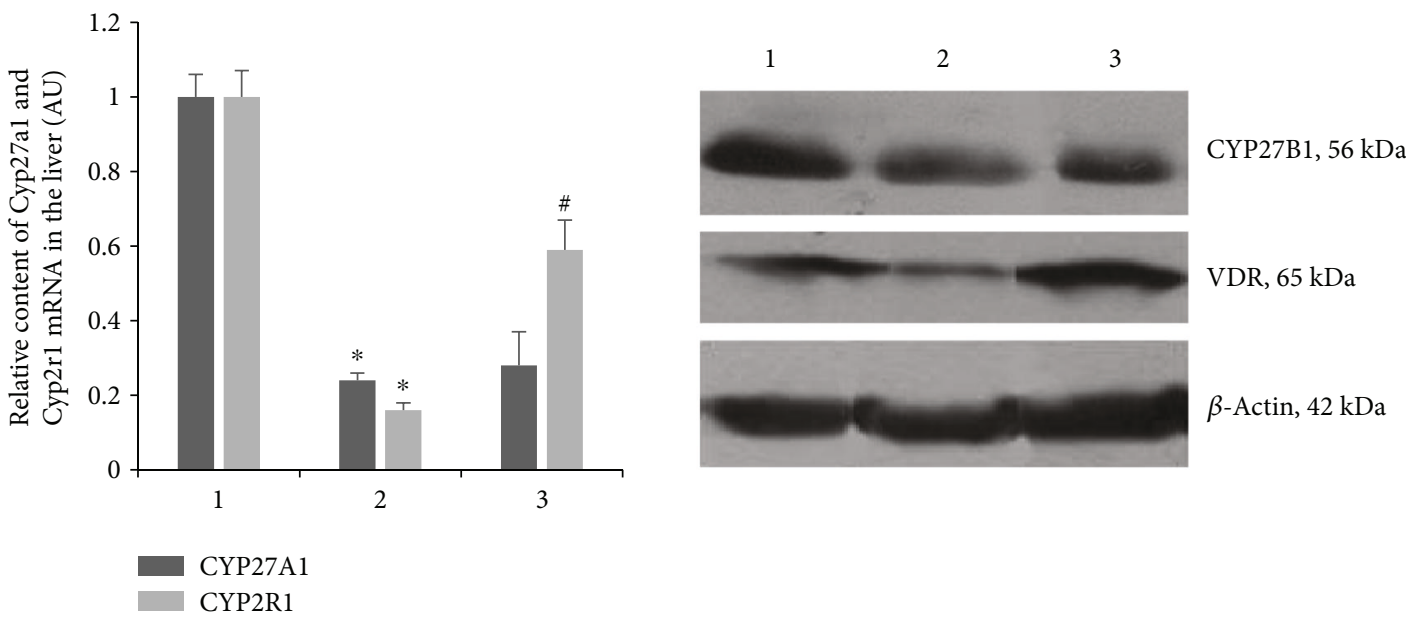

(a)

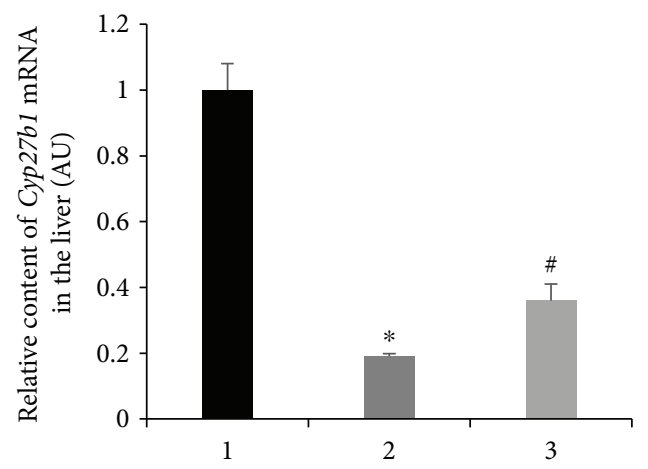

(b)

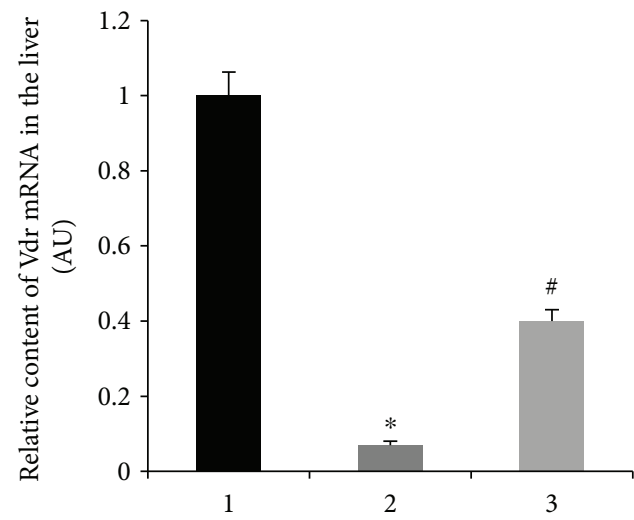

(d)

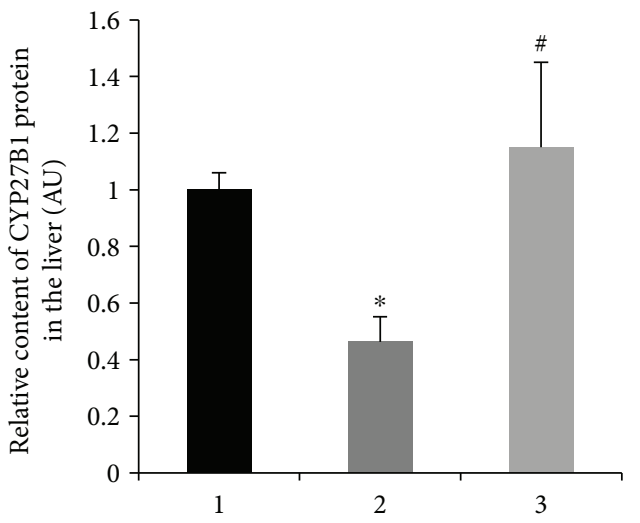

(c)

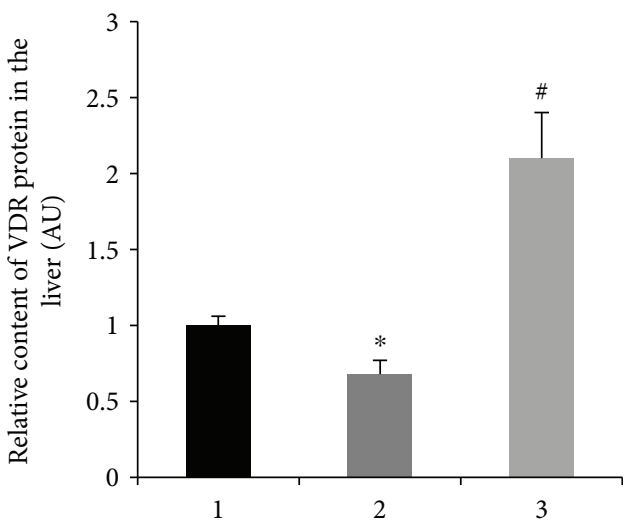

(e)

Figure 1: Transcript level (mRNA) of Cyp27a1, Cyp2r1 (a), Cyp27b1 (b), and Vdr (d) and protein abundance of CYP27B1 (c) and VDR (e) in liver tissue of diabetic rats and after vitamin $\mathrm{D}_{3}$ treatment. 1 -control; 2 -diabetic rats; and 3 -vitamin $\mathrm{D}_{3}$-treated diabetic rats. Findings are shown in representative immunoblots. Results are expressed as mean \pm SEM. ${ }^{*} p<0.05$ versus control; ${ }^{\#} p<0.05$ versus diabetes, $n=6$.

complications [10]. It was shown that calciferols have immunomodulatory effects that directly affect immune cell maturation and cytokine synthesis. On the one hand, a lack of vitamin $\mathrm{D}$ can contribute to the development of T1D, since cholecalciferol does not provide an adequate immunomodulatory effect. On the other hand, among the numerous complications of diabetes, the functions of the kidneys, liver, and many other tissues, which normally participate in vitamin D metabolism, are disrupted. Our study was performed to characterize the expression of key molecules involved in the metabolism and signaling of vitamin $\mathrm{D}$ in different tissues in experimental T1D and to assess the efficacy of cholecalciferol to correct the changes associated with diabetes. 


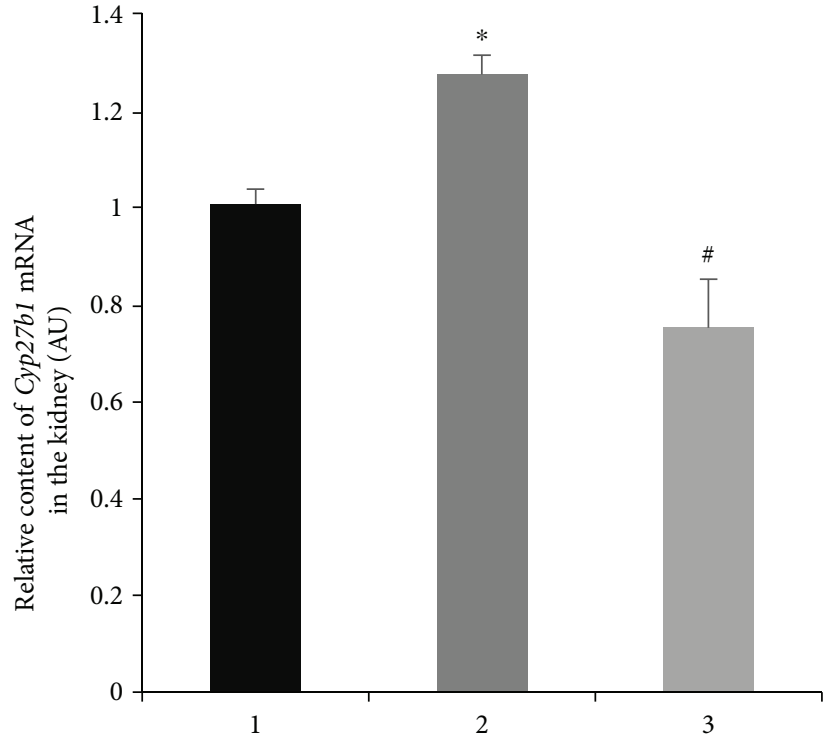

(a)

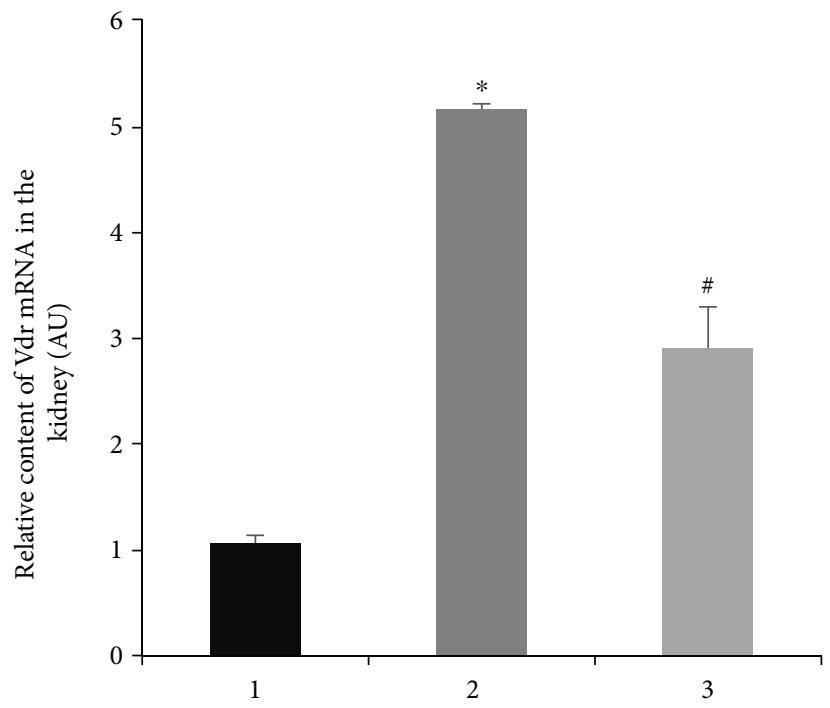

(c)

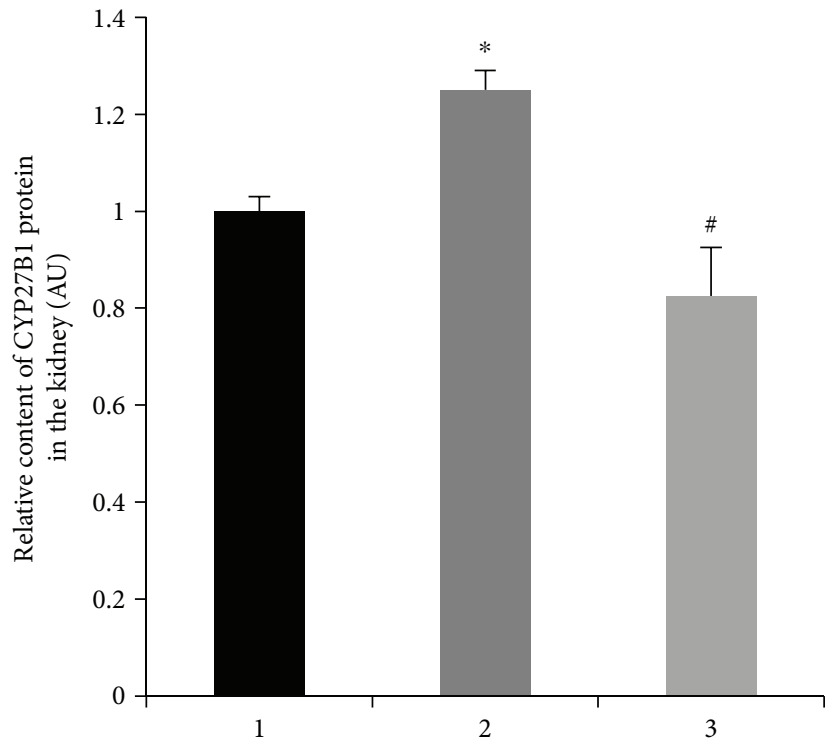

(b)

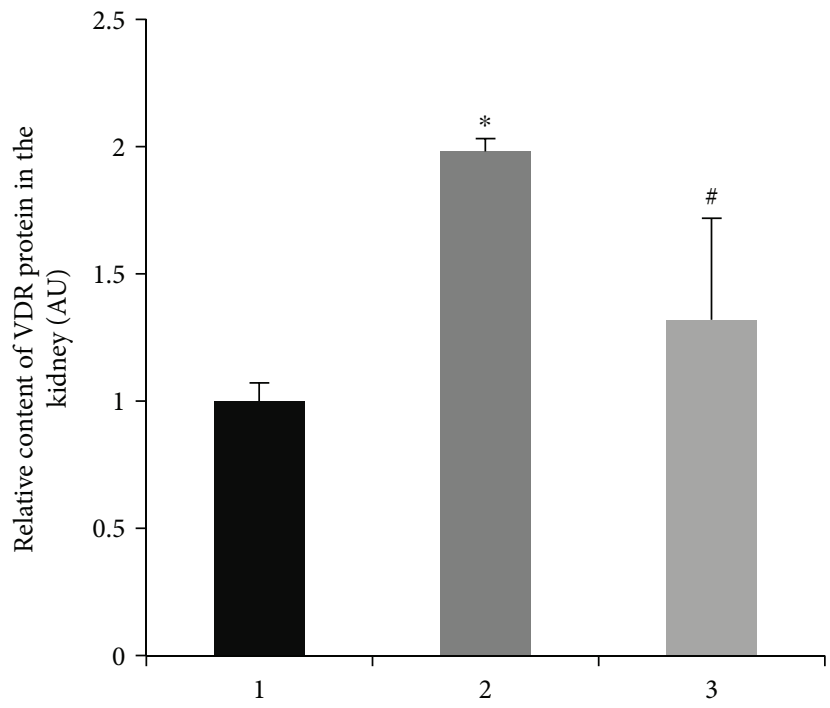

(d)
CYP27B1, $56 \mathrm{kDa}$

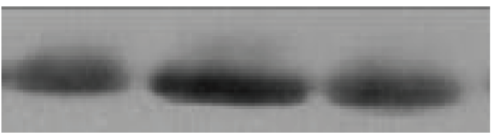

2

3

$\mathrm{VDR}, 65 \mathrm{kDa}$

$\beta$-Actin, $42 \mathrm{kDa}$

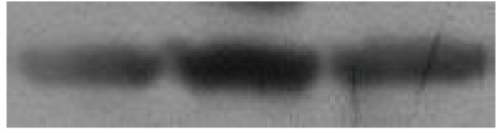

1

2

3

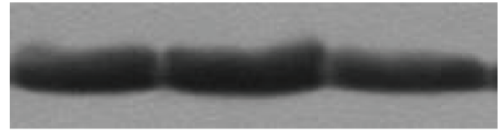

1

2

3

FIGURE 2: Transcript level (mRNA) and protein abundance of CYP27B1 (a, b) and VDR (c, d) in kidney tissue of diabetic rats and after vitamin $\mathrm{D}_{3}$ treatment. 1-control; 2-diabetic rats; and 3-vitamin $\mathrm{D}_{3}$-treated diabetic rats. Findings are shown in representative immunoblots. Results are expressed as mean \pm SEM. ${ }^{*} p<0.05$ versus control; ${ }^{*} p<0.05$ versus diabetes, $n=6$.

Vitamin D metabolism is carried out in various tissues and organs, among which the main role is assigned to the liver and kidneys. A significantly smaller endocrine role, but much more considerable in para/autocrine regulation of cellular functions, is played by extrarenal tissues [15]. The first step of vitamin D hydroxylation occurs in the liver with the help of cytochromes P450, CYP27A1 and CYP2R1 (vitamin D 25-hydroxylases). Our study demonstrated that mRNA levels of both hydroxylases diminished significantly in T1D. Vitamin $\mathrm{D}_{3}$ markedly increased the expression of Cyp2r1 without affecting Cyp27a1. Among the factors potentially contributing to the selective effect of cholecalciferol on only one of the two isoforms of hydroxylases, we should take into account the important differences in the 


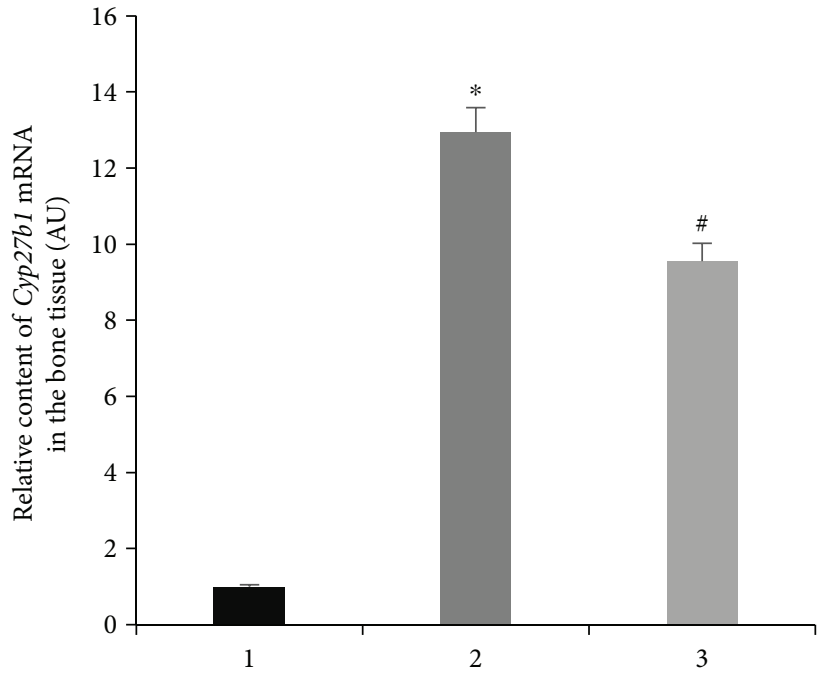

(a)

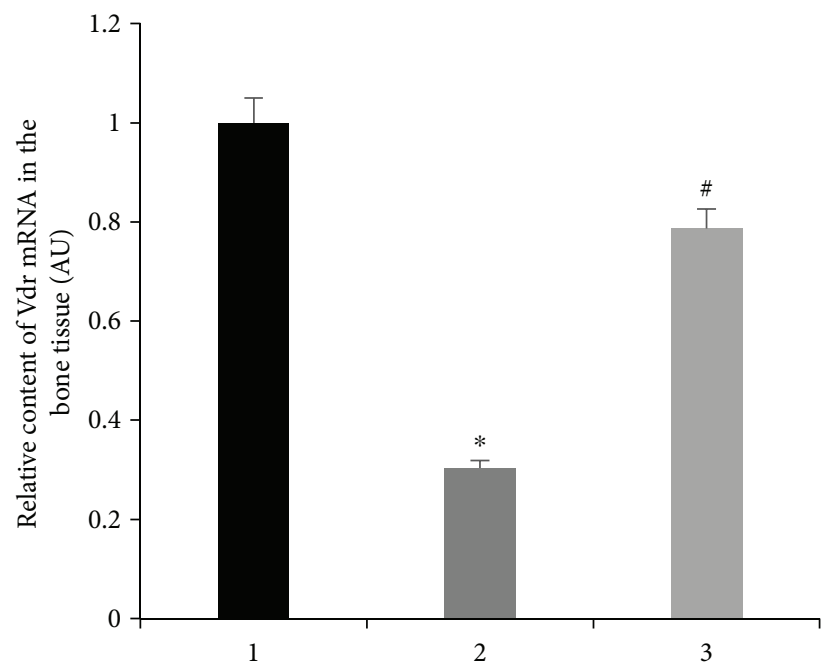

(c)

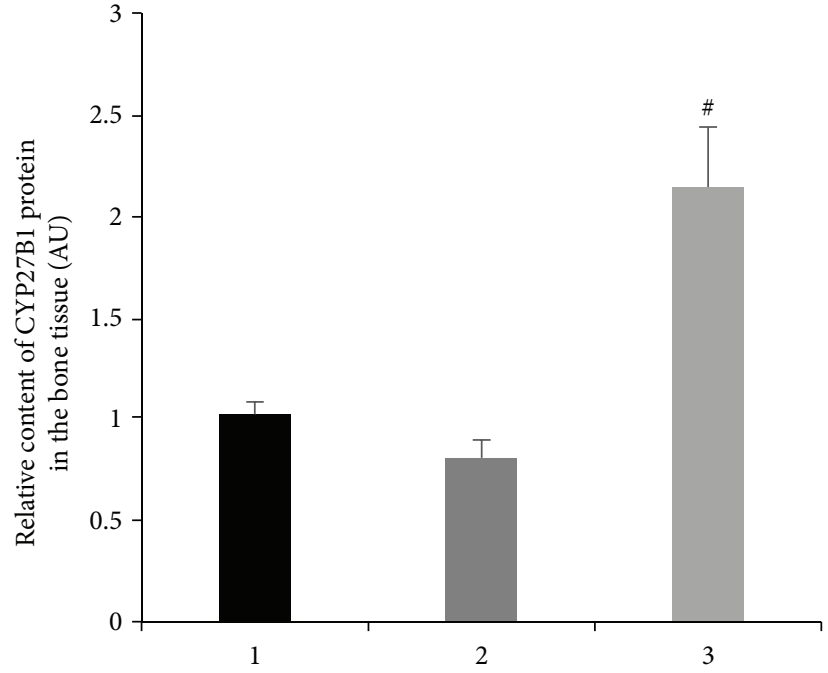

(b)

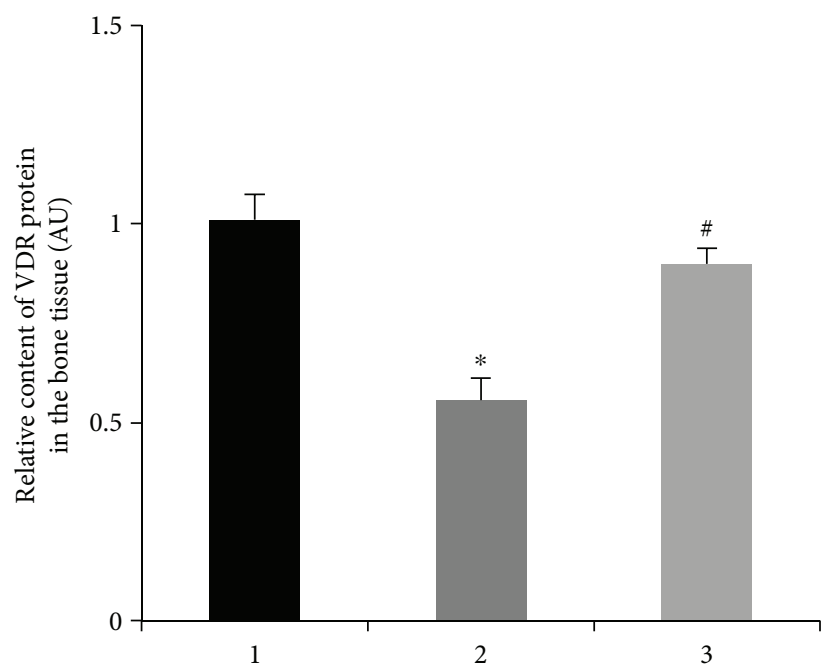

(d)
CYP27B1, $56 \mathrm{kDa}$

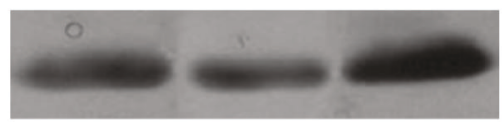

1

2

3
VDR, $65 \mathrm{kDa}$

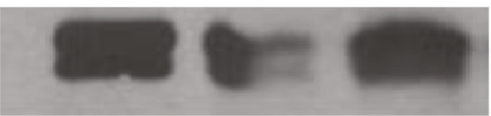

1
$\beta$-Actin, $42 \mathrm{kDa}$

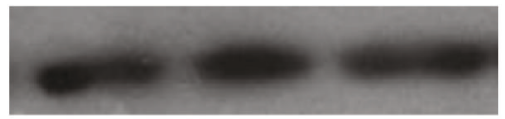

2

FIGURE 3: Transcript level (mRNA) and protein abundance of CYP27B1 (a, b) and VDR (c, d) in bone tissue of diabetic rats and after vitamin $\mathrm{D}_{3}$ treatment. 1-control; 2-diabetic rats; and 3-vitamin $\mathrm{D}_{3}$-treated diabetic rats. Findings are shown in representative immunoblots. Results are expressed as mean \pm SEM. ${ }^{*} p<0.05$ versus control; ${ }^{\#} p<0.05$ versus diabetes, $n=6$.

physiological functions that these isoforms fulfill in hepatocytes. CYP2R1 is known to be an inducible enzyme with a greater affinity for vitamin $\mathrm{D}$ and higher hydroxylase activity than CYP27A1 [16]. It can be assumed that CYP2R1, as an inducible enzyme, is able to respond promptly to cholecalciferol load in diabetes to maintain a sufficient level of $25 \mathrm{OHD}$ prohormone for the realization of its physiological functions.

Subsequently, we investigated the expression of hepatic CYP27B1 and VDR, which are responsible for the synthesis of calcitriol and its cellular signaling, that ensures the implementation of vitamin D function locally in the liver [17]. Our results demonstrated a decrease in Cyp27b1 mRNA, which was accompanied by the downregulation of its protein level. We observed similar changes with respect to the expression of VDR in diabetic liver. These findings can be supported by scientific data on the relationship between vitamin D insufficiency and declined VDR expression in hepatic steatosis [18]. Decreased expression of CYP27B1 and VDR, which we established, is most likely a consequence of vitamin D deficiency and 

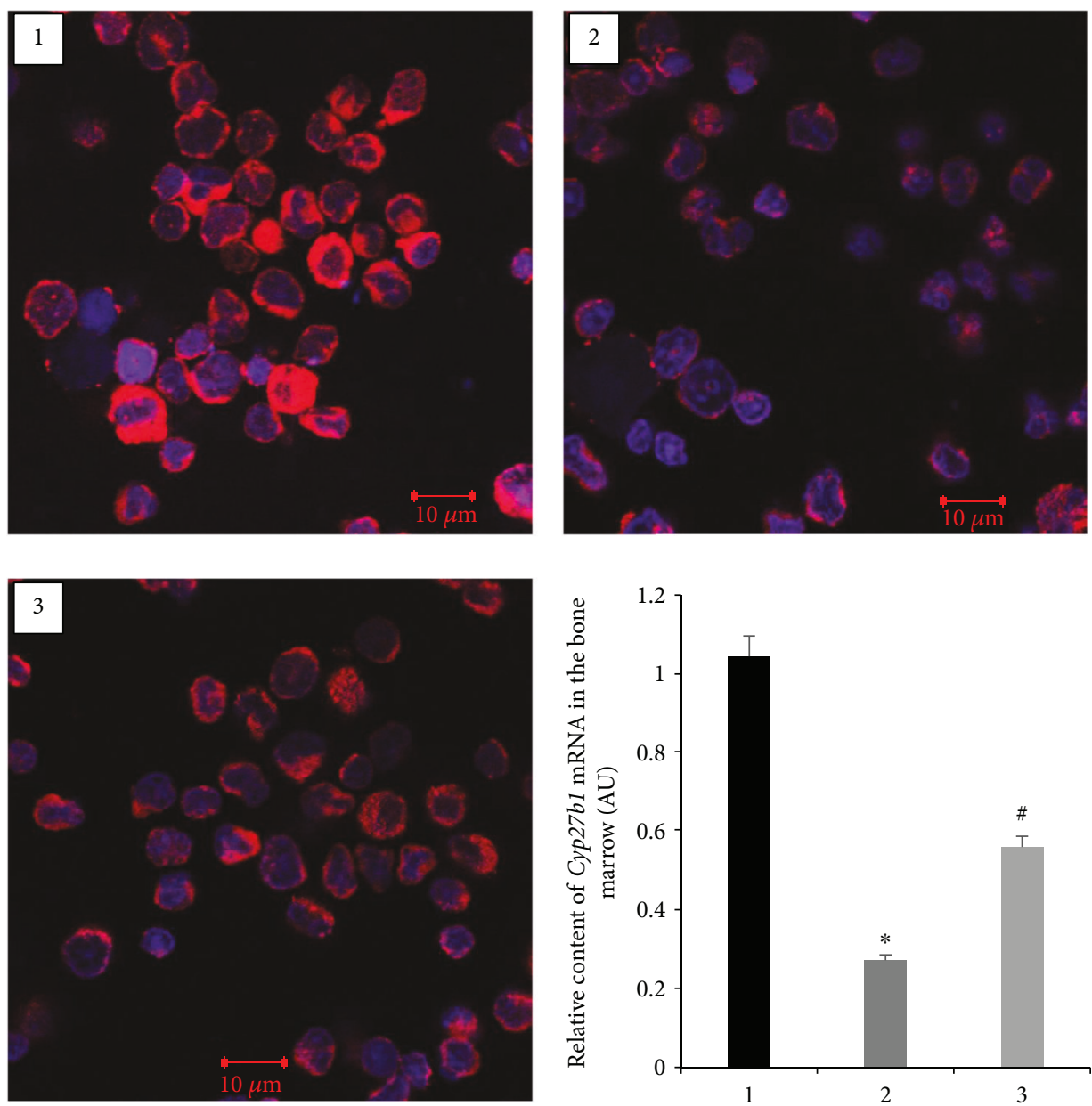

FIGURE 4: Immunofluorescence staining and transcript level (mRNA) of Cyp27b1 in the bone marrow of diabetic rats and after vitamin $\mathrm{D}_{3}$ treatment. 1-control; 2-diabetic rats; and 3-vitamin $\mathrm{D}_{3}$-treated diabetic rats. Harvested bone marrow cells were stained for CYP27B1 (red fluorescence; scale bars, $10 \mu \mathrm{m}$ ). Nuclei were counterstained with Hoechst. Results of RT-qPCR are expressed as mean \pm SEM. ${ }^{*} p<0.05$ versus control; ${ }^{*} p<0.05$ versus diabetes, $n=6$.

inadequate production of the $25 \mathrm{OHD}$ prohormone in the liver of diabetic animals. Cholecalciferol treatment significantly attenuated the harmful effect of diabetes on CYP27B1 and VDR expression in hepatic tissue, which additionally supports the association of these changes with the reduced vitamin $\mathrm{D}$ bioavailability.

It is known that Cyp27b1 gene expression in the kidneys is regulated by a number of physiologically important substances, such as circulating $\mathrm{Ca}^{2+}$ and inorganic phosphorus, parathyroid hormone, fibroblast growth factor-23, and, directly, by calcitriol $[2,8]$. According to the available data, vitamin D status negatively correlates with CYP27B1 synthesis [19]. Consistent with this, we found that a decrease in 25OHD blood level in experimental animals led, most likely, to a compensatory increase in the synthesis of both CYP27B1 mRNA and protein, and vitamin $\mathrm{D}_{3}$ administration reversed these parameters to control values. The positive effect of cholecalciferol is possibly achieved via $1,25(\mathrm{OH})_{2} \mathrm{D}_{3}$ interaction with VDR, which binds to the promoter region in the Cyp27b1 gene and controls its expression. We found that, together with the decreased availability of vitamin $\mathrm{D}$ in diabetics, there is a compensatory elevation of renal VDR synthesis. This mechanism can be explained by the assumption that at low concentrations of $25 \mathrm{OHD}$, an increase in $1 \alpha$-hydroxylase in the kidneys ensures the conversion of calcidiol at the highest possible level.

Current evidence suggests that 25OHD directly affects bone mineralization as the skeleton can be referred to a site of $1,25(\mathrm{OH})_{2} \mathrm{D}$ extrarenal synthesis [20]. Bone tissue VDRs are known to be predominantly localized on osteoblasts, while $25 \mathrm{OH}-1 \alpha$-hydroxylase can be expressed both in osteoblasts and osteoclasts. Our results revealed a diabetes-evoked decrease in bone tissue levels of VDR mRNA and protein that can reflect the impairment of osteoblast-mediated osteosynthesis. The reverse effect was observed on Cyp27b1 gene transcription, which, as we have shown, increased significantly in diabetics. WB indicated that transcriptional activation of $C y p 27 b 1$ gene expression was not sufficient for further protein synthesis of the $25 \mathrm{OH}-1 \alpha$-hydroxylase, since the CYP27B1 protein level remained unaffected. We can speculate that the difference in the synthesis of CYP27B1 mRNA and protein may indicate its distinctive expression in different types of bone tissue cells, in particular its probable blockage in osteoblasts and, conversely, activation in osteoclasts. 

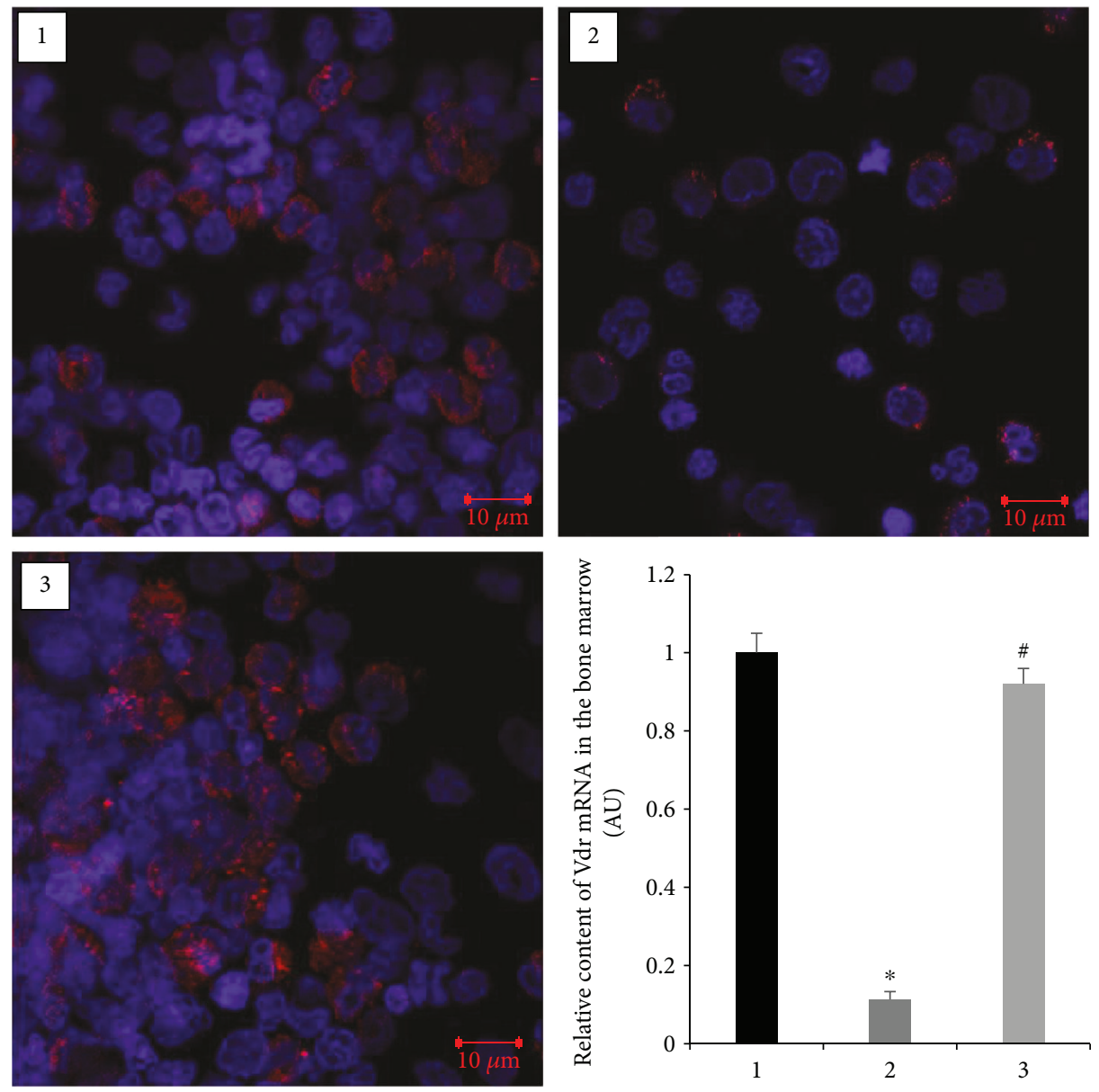

FIGURE 5: Immunofluorescence staining and transcript level (mRNA) of VDR in the bone marrow of diabetic rats and after vitamin $\mathrm{D}_{3}$ treatment. 1-control; 2-diabetic rats; and 3-vitamin $\mathrm{D}_{3}$-treated diabetic rats. Harvested bone marrow cells were stained for VDR (red fluorescence; scale bars, $10 \mu \mathrm{m}$ ). Nuclei were counterstained with Hoechst. RT-qPCR data are expressed as mean \pm SEM. ${ }^{*} p<0.05$ versus control; ${ }^{\#} p<0.05$ versus diabetes, $n=6$.

Notably, CYP27B1 expression in osteoclasts increases in direct proportion to the degree of their differentiation and activation that usually correlates with increased bone resorption [20]. The imbalance of VDRs and CYP27B1 in bone tissue from diabetic rats found in this study may reflect the changed ratio of osteoblasts to osteoclasts with a shift to the formation of active osteoclasts, which can cause bone resorption and lead to the development of secondary osteoporosis. Vitamin $\mathrm{D}_{3}$ treatment can help normalize molecular and cellular mechanisms in the coupling of bone formation to resorption, which results in restoration of osteoblast/osteoclast balance and improve bone remodeling.

Osteogenesis is a highly regulated process, in which subpopulations of bone marrow cells differentiate into mature skeletal tissues to maintain and repair the skeletons [21]. Precursors of basic bone-forming cells (osteocytes and osteoblasts) are mesenchymal stem cells localized in the bone marrow. Osteoclasts are derived from precursors of the myeloid/monocyte lineage that circulate in the blood after their formation in the bone marrow [22]. A number of research efforts indicate that changes in the differentiation and functional activity of osteoblasts play a decisive role in the occurrence of secondary osteoporosis in T1D due to hyperglycemia, generalized oxidative stress, and inflammation [23]. High glucose level induces the synthesis of proinflammatory cytokines that leads to a delay in the maturation of osteoblasts. In addition, hyperglycemia increases PPAR $\gamma$ synthesis by liver cells, which reverses the differentiation of macrophages from osteoclasts to adipocytes [24]. In keeping with the above, we established reduced levels of $V d r$ mRNA in the bone marrow cells isolated from the diabetic rats as well as decreased VDR immunofluorescence. These results may indicate a slowdown in the differentiation of bone marrow precursors, which can lead to diabetes-associated defects in osteosynthesis. Cholecalciferol was effective in correcting the revealed disturbances that confirms the possible role of vitamin D deficiency and impairments of its metabolism in the development of bone marrow cell dysfunction related to diabetes.

\section{Conclusion}

Collectively, our data indicate that T1D development causes vitamin $\mathrm{D}$ deficiency and associated vitamin D-endo/para/ 
autocrine system disruption in "classical" organs and extrarenal tissues involved in vitamin $\mathrm{D}$ metabolism and $1,25(\mathrm{OH})_{2} \mathrm{D}$ signaling. With a general decrease in the synthesis of CYP27B1 and VDR in extrarenal tissues, a compensatory elevation of their expression in the kidneys is most likely. Cholecalciferol administration as a potential hydroxylation substrate and precursor of vitamin D-hormone partially or completely attenuated diabetes-induced abnormalities in the vitamin $\mathrm{D}$-endo/para/autocrine system. Maintaining a sufficient level of vitamin $\mathrm{D}$ in diabetes contributes to the normalization of impairments in the metabolism of vitamin D and VDR-mediated cellular signaling.

\section{Conflicts of Interest}

All authors agree that they have no conflict of interests.

\section{Authors' Contributions}

Anna Mazanova and Ihor Shymanskyi contributed equally to this work.

\section{Acknowledgments}

This work was supported by a research grant from the interdisciplinary program "Molecular and Cellular Biotechnology for Medicine" (no. 32) of the National Academy of Sciences of Ukraine.

\section{References}

[1] A. Hossein-nezhad and M. F. Holick, "Vitamin D for health: a global perspective," Mayo Clinic Proceedings, vol. 88, no. 7, pp. 720-755, 2013.

[2] S. Christakos, P. Dhawan, A. Verstuyf, L. Verlinden, and G. Carmeliet, "Vitamin D: metabolism, molecular mechanism of action, and pleiotropic effects," Physiological Reviews, vol. 96, no. 1, pp. 365-408, 2016.

[3] S. Samuel and M. D. Sitrin, "Vitamin D's role in cell proliferation and differentiation," Nutrition Reviews, vol. 66, Supplement 2, pp. S116-S124, 2008.

[4] A. Zittermann and J. F. Gummert, "Nonclassical vitamin D actions," Nutrients, vol. 2, no. 4, pp. 408-425, 2010.

[5] R. Riachy, B. Vandewalle, E. Moerman et al., "1,25-Dihydroxyvitamin $\mathrm{D}_{3}$ protects human pancreatic islets against cytokineinduced apoptosis via down-regulation of the Fas receptor," Apoptosis, vol. 11, no. 2, pp. 151-159, 2006.

[6] K. C. Chiu, A. Chu, V. L. Go, and M. F. Saad, "Hypovitaminosis $\mathrm{D}$ is associated with insulin resistance and $\beta$ cell dysfunction," The American Journal of Clinical Nutrition, vol. 80, no. 6, pp. 820-825, 2004.

[7] A. W. Norman, "From vitamin D to hormone D: fundamentals of the vitamin D endocrine system essential for good health," The American Journal of Clinical Nutrition, vol. 88, no. 2, pp. 491S-499S, 2008.

[8] T. Richart, Y. Li, and J. Staessen, "Renal versus extrarenal activation of vitamin $\mathrm{D}$ in relation to atherosclerosis, arterial stiffening, and hypertension," American Journal of Hypertension, vol. 20, no. 9, pp. 1007-1015, 2007.

[9] J. D. Cooper, D. J. Smyth, N. M. Walker et al., "Inherited variation in vitamin $\mathrm{D}$ genes is associated with predisposition to autoimmune disease type 1 diabetes," Diabetes, vol. 60, no. 5, pp. 1624-1631, 2011.

[10] C. Mathieu and K. Badenhoop, "Vitamin D and type 1 diabetes mellitus: state of the art," Trends in Endocrinology \& Metabolism, vol. 16, no. 6, pp. 261-266, 2005.

[11] L. You, L. Chen, L. Pan, and J. Y. Chen, "New insights into the gene function of osteoporosis," Frontiers in Bioscience, vol. 18, no. 3, pp. 1088-1097, 2013.

[12] I. O. Shymanskyy, A. O. Mazanova, and M. M. Veliky, "Development and validation of immunoenzyme test-system for determination of 25-hydroxyvitamin D in blood serum," Biotechnologia Acta, vol. 9, no. 2, pp. 28-36, 2016.

[13] I. O. Shymanskyy, O. O. Lisakovska, A. O. Mazanova, D. O. Labudzynskyi, A. V. Khomenko, and M. M. Veliky, "Prednisolone and vitamin $\mathrm{D}_{3}$ modulate oxidative metabolism and cell death pathways in blood and bone marrow mononuclear cells," The Ukrainian Biochemical Journal, vol. 88, no. 5, pp. 38-47, 2016.

[14] J. Tuomilehto, "The emerging global epidemic of type 1 diabetes," Current Diabetes Reports, vol. 13, no. 6, pp. 795804, 2013.

[15] M. Wacker and M. Holick, "Vitamin D-effects on skeletal and extraskeletal health and the need for supplementation," Nutrients, vol. 5, no. 1, pp. 111-148, 2013.

[16] R. Shinkyo, T. Sakaki, M. Kamakura, M. Ohta, and K. Inouye, "Metabolism of vitamin D by human microsomal CYP2R1," Biochemical and Biophysical Research Communications, vol. 324, no. 1, pp. 451-457, 2004.

[17] K.-C. Chiang, C.-L. Yen, C.-N. Yeh et al., "Hepatocellular carcinoma cells express $25(\mathrm{OH}) \mathrm{D}-1 \alpha$-hydroxylase and are able to convert $25(\mathrm{OH}) \mathrm{D}$ to $1 \alpha, 25(\mathrm{OH})_{2} \mathrm{D}$, leading to the $25(\mathrm{OH}) \mathrm{D}$-induced growth inhibition," The Journal of Steroid Biochemistry and Molecular Biology, vol. 154, pp. 47-52, 2015.

[18] I. Barchetta, S. Carotti, G. Labbadia et al., "Liver vitamin D receptor, CYP2R1, and CYP27A1 expression: relationship with liver histology and vitamin $\mathrm{D}_{3}$ levels in patients with nonalcoholic steatohepatitis or hepatitis C virus," Hepatology, vol. 56, no. 6, pp. 2180-2187, 2012.

[19] H. L. Henry, "Regulation of vitamin D metabolism," Best Practice \& Research Clinical Endocrinology \& Metabolism, vol. 25, no. 4, pp. 531-541, 2011.

[20] M. Kogawa, P. H. Anderson, D. M. Findlay, H. A. Morris, and G. J. Atkins, "The metabolism of 25-(OH)vitamin $\mathrm{D}_{3}$ by osteoclasts and their precursors regulates the differentiation of osteoclasts," The Journal of Steroid Biochemistry and Molecular Biology, vol. 121, no. 1-2, pp. 277-280, 2010.

[21] F. A. Fierro, J. A. Nolta, and I. E. Adamopoulos, "Concise review: stem cells in osteoimmunology," Stem Cells, vol. 35, no. 6, pp. 1461-1467, 2017.

[22] S. S. Kohli and V. S. Kohli, "Role of RANKL-RANK/osteoprotegerin molecular complex in bone remodeling and its immunopathologic implications," Indian Journal of Endocrinology and Metabolism, vol. 5, no. 3, pp. 175-181, 2011.

[23] P. Dhaon and V. N. Shah, "Type 1 diabetes and osteoporosis: a review of literature," Indian Journal of Endocrinology and Metabolism, vol. 18, no. 2, pp. 159-165, 2014.

[24] S. Botolin and L. R. McCabe, "Inhibition of PPAR $\gamma$ prevents type I diabetic bone marrow adiposity but not bone loss," Journal of Cellular Physiology, vol. 209, no. 3, pp. 967976, 2006. 


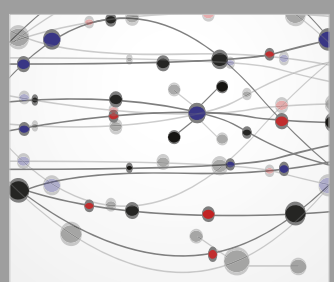

The Scientific World Journal
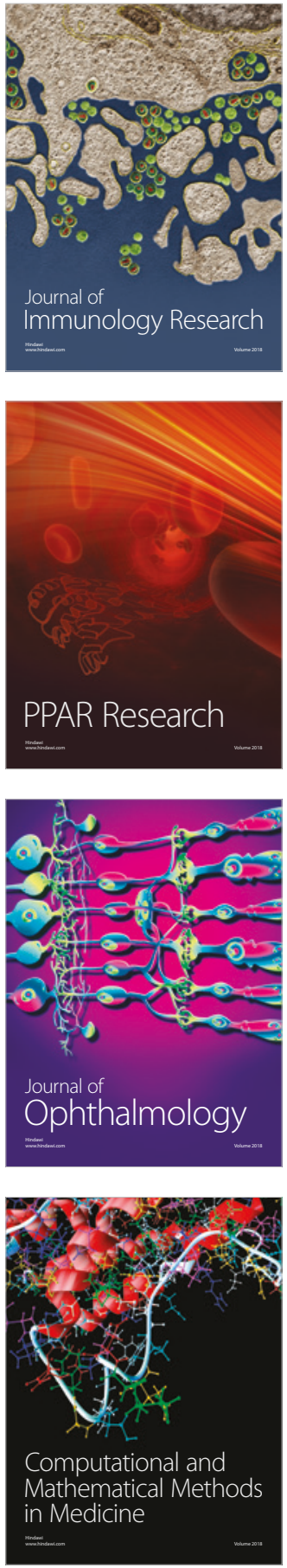

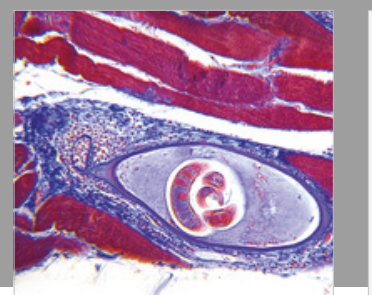

Gastroenterology Research and Practice

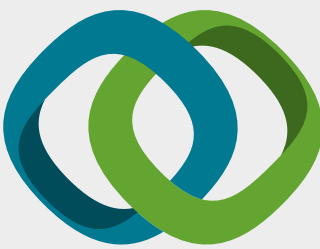

\section{Hindawi}

Submit your manuscripts at

www.hindawi.com
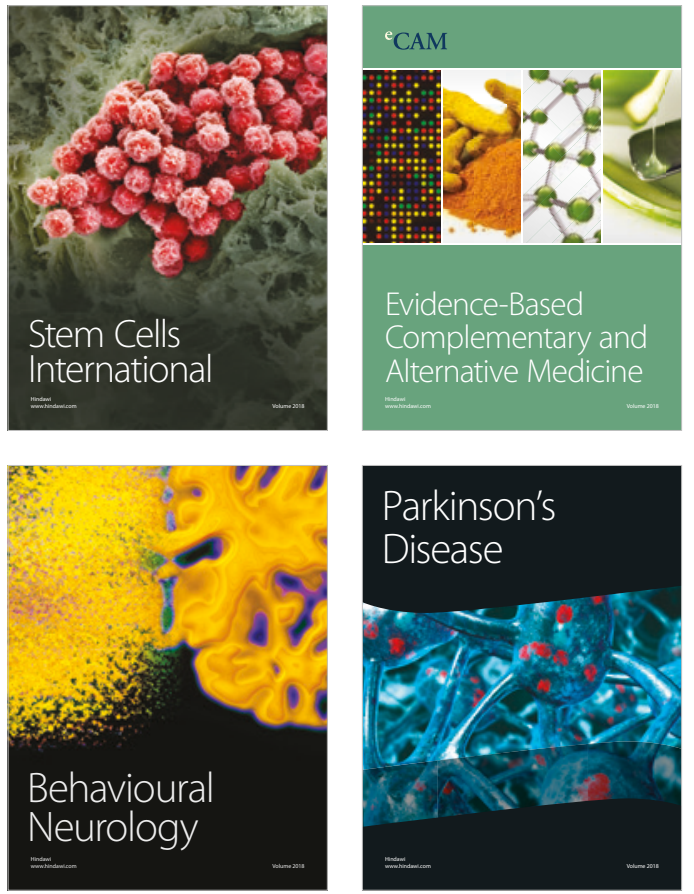

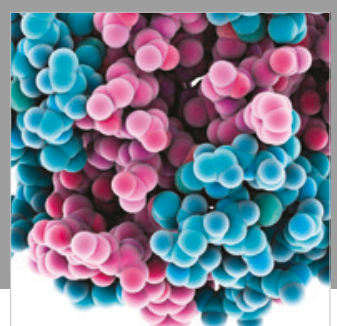

ournal of

Diabetes Research

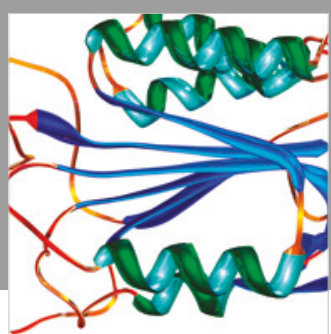

Disease Markers
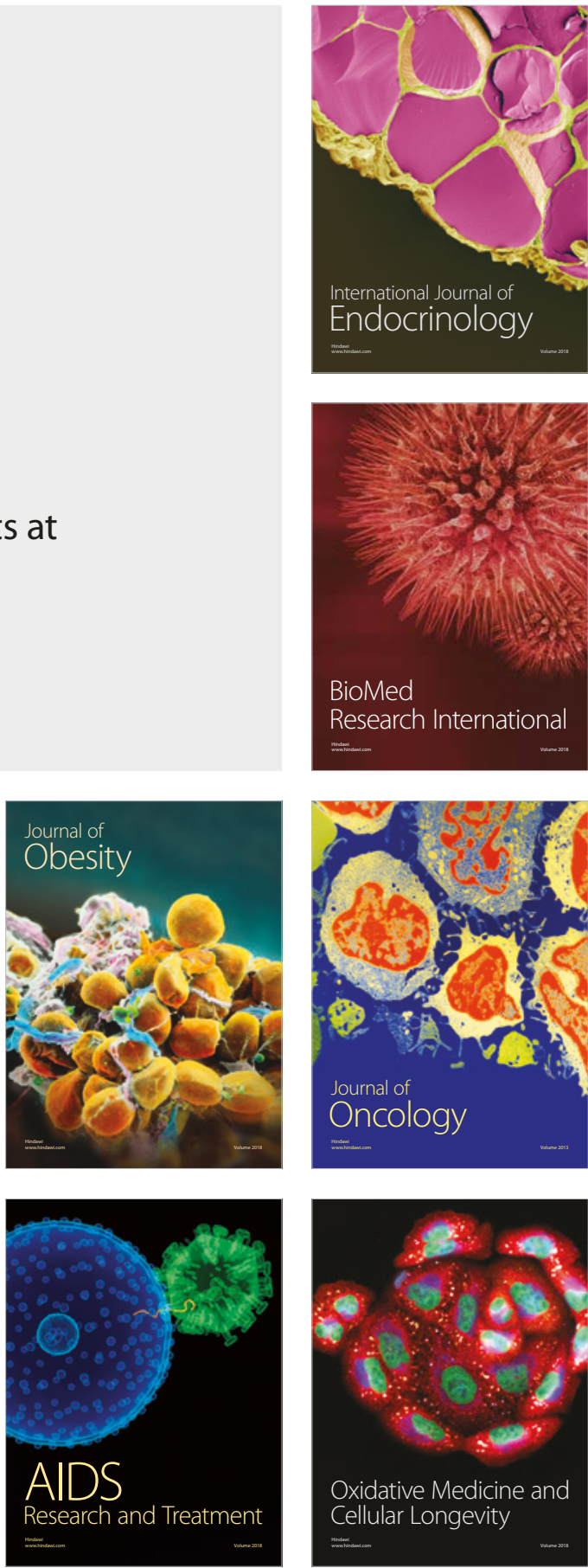